\title{
Compressibilities of Crystalline and Glassy Modifications of Selenium and Glucose ${ }^{1}$
}

\author{
Charles E. Weir
}

\begin{abstract}
Data are reported for the compression of selenium and $\alpha$-glucose in both glassy and crystalline modifications at $21^{\circ} \mathrm{C}$ between 1,000 and 10,000 atmospheres. The compressibilities of the glassy form were found to be considerably larger than those of the crystals. A transition of unknown origin was observed to occur in glassy glucose between 7,000 and 8,000 atmospheres.
\end{abstract}

\section{Introduction}

It has been observed that the expansivity, $\alpha$, of the glassy form of a given material is nearly equal to that of the crystalline form [1]. ${ }^{2}$ This agreement in expansivities appears to be the basis from which recent ideas concerning the nature of the glass transition and the changes that occur at the transition temperature, $T_{\mathrm{g}}[1,2]$ are derived. As many second-order thermodynamic quantities appear to change discontinuously at $T_{\mathrm{g}}$, this frequently has been denoted a second-order transition temperature. The expansivity is one of the quantities that changes markedly at $T_{\mathrm{g}}$, being smaller for the glassy material than for the rubbery or liquid modification that exists at temperatures above $T_{\mathrm{g}}[1,3]$.

It is believed that the isothermal compressibility, $\beta$, in analogy with expansivity, also changes discontinuously at $T_{\mathrm{g}}$ [1], but a recent report [4] indicates that most evidence for such a discontinuity in $\beta$ is inconclusive. As expansivities of crystalline and glassy forms are about equal, the question arises whether the same is true of $\beta$. Compressibility data on glasses [3] show that the compressibility is high and varies rapidly but "irresponsibly" with temperature. Data covering any appreciable pressure range in which values for glassy and crystalline modifications can be compared, however, do not appear to be available. This report describes compression measurements at $21^{\circ} \mathrm{C}$ on two materials, selenium and glucose, in glassy and crystalline modifications. A considerable difference in compressibility of the two forms was observed over the whole pressure range (maximum 10,000 atm). A reversible first-order transition of unknown origin was observed in glassy glucose at about 7,000 atm.

\section{Experimental Method}

The experimental procedure used has been described in detail [5] and will not be discussed here. The only departure from previous techniques was in the use of a steel cylinder that was open at one end, to contain the glassy glucose, and containers made of thin polyethylene film for the other materials. Both types of containers were open to the confining liquid to permit application of hydrostatic pressure

${ }_{1}$ This work was sponsored by the Office of Naval Research, Department of the Navy.

2 Figures in brackets indicate the literature references at the end of this paper to the specimens. In each case the data were corrected for the compression of the corresponding container.

Specimens were prepared as follows:

Selenium. - Commercially available selenium was placed in a thick-walled glass tube, which was then evacuated and sealed off under vacuum. The evacuated tube and its contents were heated at $400^{\circ} \mathrm{C}$ for several hours cooled in air to approximately $200^{\circ} \mathrm{C}$, and water-quenched. For glassy selenium specimens the tube was broken and the specimen removed. Such specimens exhibited the typical conchoidal fractures characteristic of glasses and were soluble in carbon disulfide [6]. For crystallization to the metallic form, the tube was not broken after quenching but reheated to $100^{\circ} \mathrm{C}$ for 2 weeks [7]. Such specimens on removal from the tube were not extractible with carbon disulfide and showed no conchoidal fractures.

Glucose.-Pure $\alpha$-dextrose was melted carefully in a glass tube. When the material was cooled, no crystallization took place, and such specimens were used for glassy-state measurements. Crystalline specimens were obtained by seeding the glassy melt and permitting crystallization to take place. Crystallization was very slow, requiring several weeks to go to completion. Cylindrical crystalline specimens were obtained by breaking the glass container.

\section{Results and Discussion}

Compression data on the glucose and selenium specimens are shown in table 1 and figures 1 and 2 . Each value given in the tables represents the average of two measurements made on different specimens. Agreement between duplicates was in general reasonably close in the last significant figure, but on occasion differences larger than the estimated reproducibility of \pm 0.0004 in $\Delta V / V_{\text {o }}$ were obtained. Negative values shown at the two lower pressures arise from the use of $2,000 \mathrm{~atm}$ as the fiducial pressure. The values between 1 and $1,000 \mathrm{~atm}$ were obtained by successive approximations and are not measured values [5]; small changes in these values will have a negligible effect on the other data. The tabular data show that the glassy material is considerably more compressible than the crystalline form. This is also shown by the graphs, in which it is observed that this higher compressibility (the slope of 


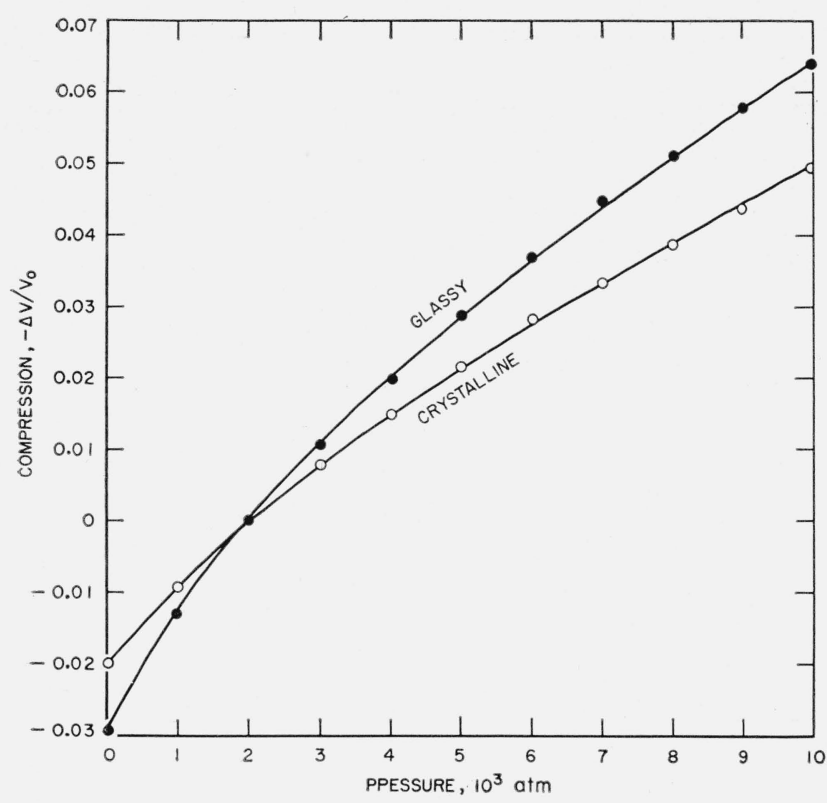

Figure 1. Compression of glassy and crystalline selenium at $21^{\circ} \mathrm{C}$.

TABLE 1. Compression of selenium and $\alpha$-glucose at $21^{\circ} \mathrm{C}$ [Compression, $-\Delta V / V_{0}$ ]

\begin{tabular}{|c|c|c|c|c|}
\hline \multirow{2}{*}{ Pressure } & \multicolumn{2}{|c|}{ Selenium } & \multicolumn{2}{|c|}{$\alpha$-Glucose } \\
\hline & Crystalline & Glassy & Crystalline & Glassy \\
\hline atm & & & & \\
\hline 10,000 & 0.0494 & 0.0643 & 0.0448 & 0.0674 \\
\hline 9,000 & .0438 & .0580 & .0392 & .0620 \\
\hline 8,000 & .0389 & .0513 & .0345 & .0562 \\
\hline 7,000 & .0334 & .0448 & .0296 & .0487 \\
\hline 6,000 & .0284 & .0370 & .0240 & .0404 \\
\hline 5,000 & .0219 & .0288 & .0188 & .0318 \\
\hline 4,000 & .0151 & .0201 & .0130 & .0222 \\
\hline 3,000 & .0079 & .0106 & .0064 & .0115 \\
\hline 2,000 & .0000 & .0000 & .0000 & .0000 \\
\hline $1,0 \mathrm{G0}$ & -.0093 & -.0130 & -.0074 & -.0128 \\
\hline 1 & -.0200 & -.0290 & -.0150 & -.0250 \\
\hline
\end{tabular}

the curve) is not confined to the lower pressures but extends over the whole pressure range.

On the basis of present concepts $[1,2,3]$, the glassy state contains essentially a frozen-in, liquidlike structure of such high viscosity that thermal readjustments are not possible in experimentally realizable time intervals. Therefore, the higher compressibility of the glass is to be anticipated. The compressibility of liquids is in general considerably greater than that of crystalline solids [8]. The difference is probably to be attributed to both the greater "free" volume of the liquid and a less orderly structure, with correspondingly less well-organized intermolecular forces. A glassy solid will then contain at least a greater free volume than the corresponding crystalline form, and its compressibility, therefore, will be the larger. In all probability,

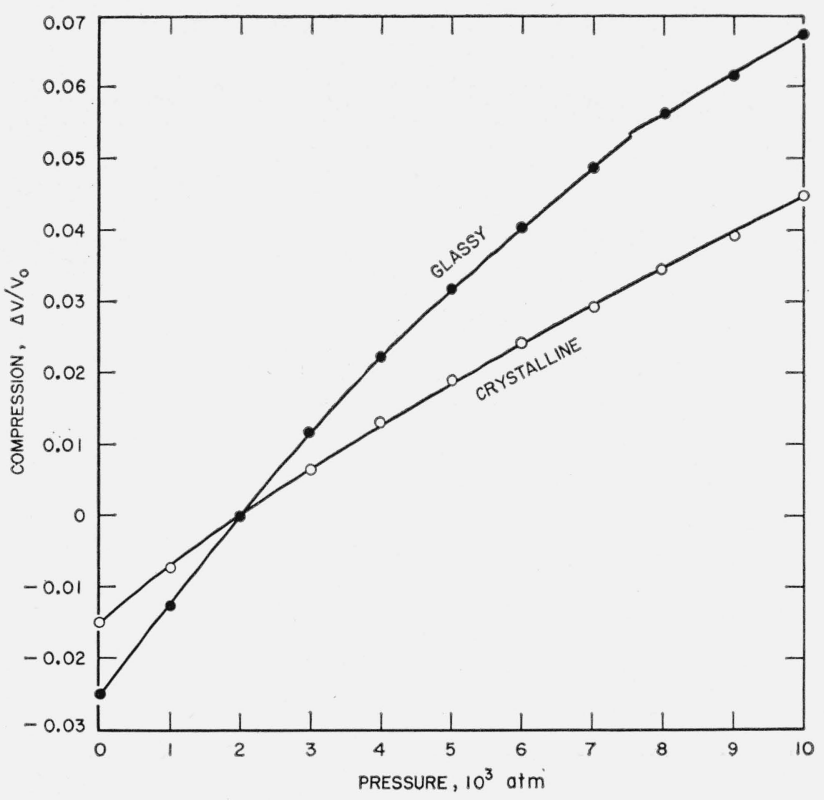

Figure 2. Compression of glassy and crystalline $\alpha$-glucose at $21^{\circ} \mathrm{C}$.

similar considerations apply to the intermolecular forces, which Bridgman [8] believes are intimately involved in this pressure range. In confirmation of this, a sample calculation shows that at 10,000 atm the specific volume of the glucose glass is considerably less than that of the crystalline material at 1 atm, yet its compressibility appears to be comparable to that of the crystals at $1 \mathrm{~atm}$.

In figure 2 a discontinuity is shown between 7,000 and $8,000 \mathrm{~atm}$. This is a reversible transition apparently involving a small discontinuity in volume and an associated latent heat. Several experiments were performed to establish the existence and reversibility of this transition, and a brief description of the phenomena involved may be of interest.

In these experiments, measurements are made on decompression [5], with the pressure being reduced in 1,050-atm steps. With attainment of thermal equilibrium after decompression, the pressure is raised again as closely as possible to the desired value. This process is followed to obtain reproducible motion of packing washers, etc. On decompression, the contents of the vessel are cooled, and a time interval ensues before thermal equilibrium is reattained. During this interval, heat is absorbed by the vessel and its contents and, as a result, the pressure rises. This "pressure regain" depends on factors such as pressure, compressibility of specimens, rate of decompression, etc., but for closely controlled conditions does not vary to a large degree. In these experiments a regain of approximately 15 to $20 \mathrm{~atm}$ is the rule. Any process that occurs on decompression involving appreciable thermal effects will cause an anomalous pressure regain and is readily noted because the pressure measurement is 
quite sensitive. In these experiments the pressure regain in the interval noted was 2 to 3 times as great as those observed at higher and lower pressures, indicating a latent-heat phenomenon in the interval. This is shown in figure 2 by the discontinuity in volume, although no effort was made either to fix its pressure accurately or to measure the amount of discontinuity.

The phenomenon described cannot represent a crystallization of the glassy form for three reasons: no crystals are visible after a compression measurement; the process is reversible; and densities of specimens agreed to better than 1 part in 10,000 before and after compression. No explanation for the processes involved will be offered. It may be noted that the product formed at higher pressures is shown by figure 2 to be considerably less compressible than its low-pressure modification. A statistical study of the experimental data indicates that this effect may be real, but a definite conclusion cannot be made with certainty.

Previous data have been reported for the compression of these materials. Bridgman [9] has reported data on selenium (Se) to $50,000 \mathrm{~kg} / \mathrm{cm}^{2}$, which were apparently made on glassy Se. However, Bridgman [9] reports that in calculations the density of Se used was probably too high. Data for glassy Se reported here are in only fair agreement with Bridgman's data. Tammann and Jellinghaus [10] reported data for glassy Se to a maximum pressure of approximately $2,000 \mathrm{~atm}$. Their measured value of $-\Delta V / V_{0}$ is approximately 0.039 at this pressure as compared with the 0.029 arrived at here and a graphical value of approximately 0.027 obtained from Bridgman's data [9]. Bridgman has also reported values for "dextrose" (d-glucose) [11] that are somewhat smaller than those obtained here. This difference may only be apparent however, as Bridgman [11] notes that his values are probably slightly too low, and it is also possible that his measurements were made on anhydrous material. In the measurements made here, the volume of the specimen was measured after compression, and it alone determined $V_{0}$. Densities calculated from this volume were lower than accepted values, probably as a result of occluded voids. This in no way affects the validity of the value of $V_{0}$.

\section{References}

[1] W. Kauzmann, Chem. Rev. 43, 219 (1948).

[2] R. O. Davies and G. O. Jones, Proc. Roy. Soc. 21\%, 26 (1953).

[3] G. W. Morey, The properties of glass (Reinhold Publishing Corp., New York, N. Y., 1938).

[4] C. E. Weir, J. Research NBS 50, 311 (1953) RP2420.

[5] C. E. Weir, J. Research NBS 45, 468 (1950) RP2160.

[6] J. W. Mellor, Comprehensive treatise on inorganic and theoretical chemistry, vol. X (Longmans, Green, and Co., New York, N. Y., 1930).

[7] S. R. Das and K. Das Gupta, Nature 143, 165 (1939).

[8] P. W. Bridgman, The physics of high pressure (G. Bell \& Sons, Ltd., London, 1949).

[9] P. W. Bridgman, Proc. Am. Acad. Arts Sci. \%4, 21 (1940).

[10] G. Tammann and W. Jellinghaus, Ann. Physik 394, 269 (1929)

[11] P. W. Bridgman, Proc. Am. Acad. Arts Sci. 76, 9 (1945).

Washington, December 8, 1953. 